\title{
Research on Fault Prediction of Electronic DeviceBased on Subjection Function
}

\author{
ZHAO Yugang \\ High_tech Institute, FanGong_ting South Street on the 12th, QingZhou, China \\ zhaoyugang z yg@sina.com
}

\begin{abstract}
Keywords: Fault prediction; Fault mechanism; Subjection function; Feature parameter; Certainty factor.

Abstract. In view of the current electronic equipment modular direction development, timely understanding of electronic equipment running status, to prevent failures and shorten the maintenance time, adopt the method of fuzzy membership function, and to quantify the fuzzy state of the equipment, introducing the credibility factor and the fusion algorithm to deal with the above data, according to the automatic test equipment (ATE) measured data and expert experience, the method are verified.The experiments show that this method is feasible and effective, for the fault of electronic equipment has high forecast accuracy.
\end{abstract}

\section{Introduction}

Modern electronic equipment is highly intelligent, complicated and modularized with continuous development of science and technology. All modules are mutually coupled and interacted, and therefore it is difficult to describe the working condition thereof by a precise mathematical model. If effective fault prediction can be implemented on electronic equipment before equipment fault, a lot of maintenance costs can be saved for equipment. Electronic equipment fault prediction has the following difficulties [1]: (1) it is difficult to establish corresponding prediction model. (2) Models are mutually coupled and interacted for correlating signals. (3) It is difficult to unify different electronic equipment fault prediction methods. (4) One fault is caused many reasons, and one reason can lead to many faults. Membership function is a concept in fuzzy theory. It reflects the mapping relationship between variable in discourse domain and elements in fuzzy set. Since electronic device fault probability is within 1-0 closed interval. In addition, the characteristic parameter value and equipment fault probability belong to one mapping. Therefore, the relationship between parameters and equipment faults can be well reflected by selecting membership function rationally.

In the paper, the working principle of some radio station is analyzed firstly. On the basis, ATE equipment test data and expert experience are combined for selecting fault prediction characteristic parameters. Since operation characteristic parameters of all existing modules directly affect operation status of corresponding module, the module fault probability can be predicted according to parameters and established membership relationship.

\section{Selection of electronic equipment fault prediction parameters}

\section{Selection of fault prediction parameters based on signal flow chart}

Signal flow chart reflects the corresponding relationship among all parts and dependency among signals during equipment operation. Signal flow chart clearly shows the corresponding relationship between fault prediction characteristic parameters and electronic equipment replaceable modules. It is supposed that a system is composed of four modules, namely $\mathrm{m} 1, \mathrm{~m} 2, \mathrm{~m} 3$, and $\mathrm{m} 4$, the module parameters for reflecting system and module state mainly include the follows: s1, s2, s3, s4, s5, s6, s7, s8, s9, s10 and s11. The signal flow chart is shown in figure 1. Wherein, T1, T2, T3, T4, T5 and T6 belong to outward test ports. Fault prediction matrix of the system can be established according to the signal flow chart as shown in table 1 , wherein all elements refer to whether module states can be reflected by fault state characteristic parameters or not [2]. 


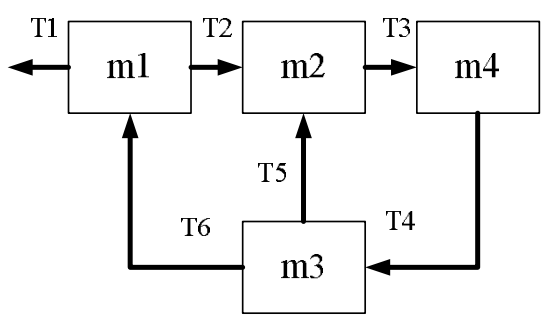

Figure 1 System signal flow chart

Table 1 Fault prediction matrix

\begin{tabular}{cccccccccccc}
\hline Test port & & & \multicolumn{1}{c}{$\mathrm{T} 1$} & $\mathrm{~T} 2$ & $\mathrm{~T} 3$ & $\mathrm{~T} 4$ & $\mathrm{~T} 5$ & $\mathrm{~T} 6$ & & & \\
\hline Main parameter & $\mathrm{s} 1$ & $\mathrm{~s} 2$ & $\mathrm{~s} 3$ & $\mathrm{~s} 4$ & $\mathrm{~s} 5$ & $\mathrm{~s} 6$ & $\mathrm{~s} 7$ & $\mathrm{~s} 8$ & $\mathrm{~s} 9$ & $\mathrm{~s} 10$ & $\mathrm{~s} 11$ \\
Module m1 & 1 & 1 & 0 & 0 & 0 & 1 & 1 & 1 & 0 & 0 & 1 \\
Module m2 & 1 & 0 & 1 & 0 & 1 & 0 & 0 & 0 & 1 & 1 & 0 \\
Module m3 & 0 & 0 & 0 & 0 & 1 & 1 & 0 & 0 & 0 & 1 & 1 \\
Module m4 & 1 & 1 & 0 & 1 & 0 & 1 & 0 & 1 & 1 & 0 & 0 \\
\hline
\end{tabular}

\section{Fault prediction parameter selection according to equipment function structure}

The working principle of equipment should be fully comprehended, and various parameters affecting normal operation of all modules can be analyzed comprehensively and accurately on one hand, a lot of monitoring data and expert experience are required in order to complete selection of fault prediction characteristic parameters under equipment function structure.

Next, the incentive module of one radio station is adopted for analysis. The ratio station has two working modes, namely conventional mode and anti-interference mode. The conventional working mode is divided into two working states, namely amplitude modulation (AM) and frequency modulation (FM); the anti-interference working mode is also divided into two working modes, namely spread spectrum (DS) and frequency hopping (DS+FH). The same module has different influence parameters under different working modes. Main characteristic parameters for reflecting incentive module operation in radio station equipment are obtained according to the analysis on the working principle of the radio station, data provided by ATE equipment and expert experience under conventional amplitude modulation (AM) working mode: UUT transmitting end current, frequency accuracy, frequency error, transmission power, AM modulation factor, AM modulation distortion, etc.

The above selected characteristic parameters can directly reflect the operation state of the module, namely characteristic parameter value reflects the operation state of the module. When the equipment is operated under healthy state, various characteristic parameters are within normal range, the health condition is continuous declining, and various prediction characteristic parameters also can be beyond the normal scope slowly due to component aging in the equipment operation process and other reasons. Equipment enters the sub-health state under the condition. Once equipment characteristic parameters are higher than the permissible value, equipment fault can be produced under general condition. The so-called sub-health state refers to meta-stable equipment configuration and performance with bidirectional transformation features to health state and fault state. Their corresponding characteristic parameter values are between permissible value and upper (lower) limit. The corresponding relationship between the characteristic parameter value and equipment state can be embodied through membership function.

\section{Membership function}

The concept of membership function was proposed by Prof. L.A.Zadeh in 1965 for realizing the purpose of quantitatively describing fuzzy object. $U$ is set as discourse domain (research scope), one fuzzy set $A$ on $U$ is represented by one real function on $U$. 


$$
\begin{aligned}
\mu_{A}: & \rightarrow[0,1] \\
& x \text { a } \mu_{A}(x)
\end{aligned}
$$

Its definition ${ }^{[3]}$ is shown as follows: function value $\mu_{A}(x)$ is called the membership degree of $\mathrm{x}$ to A aiming at $\mathrm{x} \in \mathrm{U}$, and function $\mu_{A}$ is called membership function of A. Membership degree $\mu_{A}(x)$ is closer to $1, \mathrm{x}$ has higher membership to $\mathrm{A}, \mu_{A}(x)$ is closer to $0, \mathrm{x}$ has lower membership to $\mathrm{A}$. The membership function $\mu_{A}(x)$ with value in interval 0,1 represents that $\mathrm{x}$ has lower membership to A.

\section{Determination method of membership function}

It is very important choose membership function properly in the electronic equipment fault prediction. It is related to whether currency fuzzy state is appropriately quantified or not. Since there is no uniform method in selecting membership function, different engineering personnel may select different membership functions. Even so, as long as current fuzzy state can be reflected correctly, they are essentially consistent though the forms are different. The following methods are generally adopted in the fault prediction field [4]: fuzzy statistics method, typical function method, Daniel expert determination method with conviction, etc. Daniel expert determination method mainly aims at establishing membership function. Concrete value is given according to personal experience, especially expert experience, and membership functions are constantly corrected. The method is more suitable for the condition with discrete and limited variable in the discourse domain. In the paper, the method is adopted during selection of membership function.

\section{Determination of electronic equipment membership function}

Membership function curve is defined as figure 2 according to determination method of membership function and nature of characteristic parameter corresponding module. When all test parameters of incentive unit in one communication radio station are tested, the test values should be determined. When fault prediction parameter $\mathrm{x} \in\left(\mathrm{x} 1^{\prime}, \mathrm{x} 1\right)$, the equipment is operated normally. When fault prediction characteristic parameter $\mathrm{x} \in(\mathrm{x} 1, \mathrm{x} 2) \cup\left(\mathrm{x} 2^{\prime}, \mathrm{x} 1^{\prime}\right)$, and characteristic parameter value is closer to the edge value, the possibility of fault will be increased gradually; When fault prediction characteristic parameter $\mathrm{x} \in\left(-\infty, \mathrm{x} 1^{\prime}\right) \cup(\mathrm{x} 1,+\infty)$, the fault state can be formed, wherein $\mathrm{x} 1{ }^{\prime}$ and $\mathrm{x} 1$ are upper and lower limits, and $\times 2$ ' and $\times 2$ are characteristic parameter permissible values.

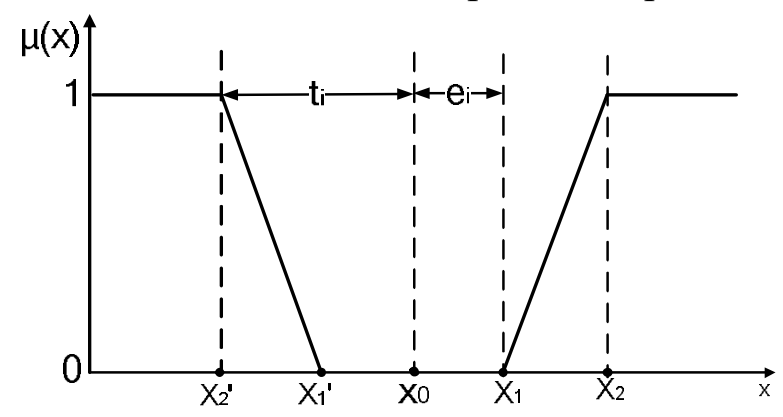

Figure 2 Membership function distribution

The membership function expression ${ }^{[5]}$ is shown as follows according to the above analysis and expert experience:

$$
u(x)=\left\{\begin{array}{cc}
1 & x_{m} \leq x_{0}-t_{i} \\
-\alpha \frac{x_{m}-x_{0}+e_{i}}{t_{i}-e_{i}} & x_{0}-t_{i}<x_{m}<x_{0}-e_{i} \\
0 & x_{0}-e_{i}<x_{m} \leq x_{0}+e_{i} \\
\alpha \frac{x_{m}-x_{0}-e_{i}}{t_{i}-e_{i}} & x_{0}+e_{i}<x_{m} \leq x_{0}+t_{i} \\
1 & x_{m}>x_{0}+t_{i}
\end{array}\right.
$$


In the formula, $x_{m}$ is the measured value of actual characteristic parameters. $x_{0}$ belongs to standard value of the measured element characteristic parameter during normal work of circuit. $t_{i}$ refers to limit deviation, $e_{i}$ refers to normal transformation scope; $u(x)$ refers to membership function belonging to fault of the fault prediction element; $\alpha$ refers to correction coefficient.

\section{Fault prediction method based on membership function}

\section{Corresponding relationship of membership degree and fault probability of measured components}

All fault prediction characteristic parameters related to incentive modules can be represented by membership function relational expression (2) according to analysis on communication radio station working principle and expert experience, namely membership value is defined as fault probability. It is assumed that membership for triggering fault parameters is $\mathrm{u}(\mathrm{i})$ respectively, corresponding fault probability is $p(j)$ respectively, then $u(i)=m \cdot p(j)$, wherein $i, j=1,2 \ldots$, and $m=1$ is set here.

\section{Establishment of the early warning value}

Fault prediction mainly depends on whether the characteristic signal is located in the allowable normal fluctuation scope or not. Equipment fault has significant representation that characteristic parameter value exceeds the permissible value. When some characteristic parameters are slightly more than permissible value, such as power, normal use of equipment may not be affected. However, some characteristic parameters, such as voltage, current, etc., are not more than the permissible value, otherwise equipment faults can be caused, and the equipment can be burned. We need to establish corresponding warning value (threshold) aiming at characteristic parameters which can lead to fault or trigger serious fault easily. It is assumed that the measured value of actual characteristic parameter is $\mathrm{x}$, its membership function $\mathrm{u}(\mathrm{x})$ meets formula (1). Meanwhile, it is required that $\mathrm{x}$ can send out early warning when the following relation expression is met.

$$
\left\{\begin{array}{l}
x \in\left(-\infty, x_{2}^{\prime}\right) \cup\left(x_{2},+\infty\right) \\
x \in\left(x_{2}, x_{1}^{\prime}\right) n\left|\frac{x^{-}-x_{2}}{x_{2}}\right| \leqslant 0.05 \\
x \in\left(x_{1}, x_{2}\right) n\left|\frac{x^{-} x_{2}}{x_{2}}\right| \leqslant 0.05
\end{array}\right.
$$

\section{Introduction of credibility factor}

Most faults are caused by changes in one characteristic parameter, and few faults are caused by other characteristic parameter due to uncertainty factors and influence of characteristic parameter characteristic during fault prediction. We adopt credibility factor [6] to solve the uncertainty. The credibility factor can be converted into the weight of fault conditions, and thereby the prediction results can be more consistent with actual condition.

Credibility factor is represented by the ratio between frequency of faults belonging to one characteristic parameter category and total fault frequency. Its calculation formula is shown as follows: fault characteristic parameters of the total number of ratios. Its computation formula is as follows: . The value range is $[0,1]$, the value is closer to 1 , and it is obvious that the fault in the category has higher probability; On the contrary, the value is closer to 0 , it is obvious that that the fault in the category has lower probability. The introduction of credibility factor represents different importance degrees of all characteristic parameters in the process of fault formation. Its structure is shown in figure 3. 


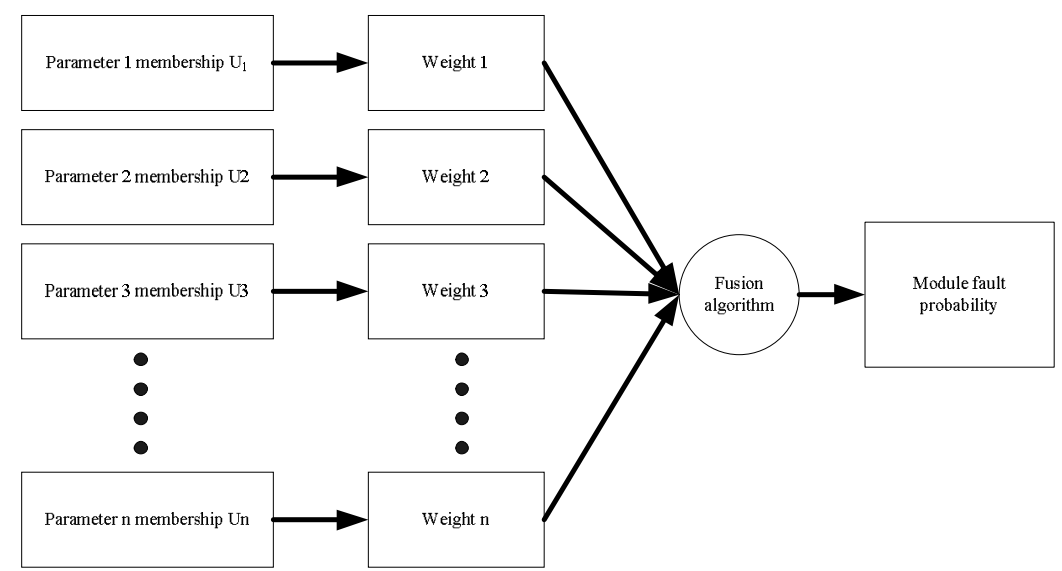

Figure 3 Fault prediction model with introduction of credibility factor (weight)

\section{Selection of fusion algorithm}

Selection of fusion algorithm has more important influence on the accuracy of prediction results, and common algorithmic rules [7] include the follows: 1) Main factor outstanding type; (2) Weighted average type; (3) comprehensive restriction type; (4) minimum upper bound type; (5) equilibrium mean type;

Main factor outstanding type not only highlights main factors, but also takes into account other factors. Therefore, main factor outstanding type is selected as fusion algorithm in the paper. The algorithm rules are shown as follows:

$$
b_{j}=\underset{i=1}{\stackrel{m}{\vee}}\left(a_{i} \cdot r_{i j}\right) \quad(j=1,2, \ldots, n)
$$

In the formula, $\bullet$ refers to common real number multiplication, refers to maximum operation.

\section{Fault prediction steps}

(1) Select characteristic parameters according to analysis of equipment working principle and expert experience;

(2) Choose appropriate membership functions;

(3) Implemented weighted average on characteristic parameters, and seek actual measured values of characteristic parameters;

(4) Compare actual measured value and set threshold value, and determine whether alarm is sent or not;

(5) Seek the probability value of faults corresponding to all characteristic parameters by using membership function;

(6) Integrate all probability values to obtain module fault probability.

4. Fault prediction instance

All important key signals should be obtained in order to achieve accurate fault prediction. In fact, it's impossible for the complex electronic equipment to obtain all signals. Characteristic parameters of incentive module working state and a part of monitoring data (AM mode $\mathrm{C}$ band) can be represented according to long-term real-time detection of ATE equipment on some communication equipment, please see table 2 .

Table 2 Characteristic signals and a part of actual

\begin{tabular}{|c|c|c|c|c|c|c|c|c|c|c|}
\hline No & Test item & $\begin{array}{l}\text { Uppe } \\
\text { r limit }\end{array}$ & $\begin{array}{l}\text { Lower } \\
\text { limit }\end{array}$ & $\begin{array}{c}\text { Allowable value } \\
\text { (upper } \\
\text { limit/lower limit) }\end{array}$ & Threshold & $\begin{array}{c}\text { Actual } \\
\text { parameter } \\
\text { value }\end{array}$ & $\begin{array}{l}\text { Early-war } \\
\text { ning or not }\end{array}$ & Membership & $\begin{array}{l}\text { Credibility } \\
\text { factor }\end{array}$ & $\begin{array}{c}\text { Fault } \\
\text { probability } \\
(\%)\end{array}$ \\
\hline 1 & $\begin{array}{c}\text { UUT } \\
\text { transmittin } \\
\mathrm{g} \text { end } \\
\text { current }(\mathrm{A})\end{array}$ & 10 & 4.0 & $10.3 / 2.8$ & 10.25 & 10.237 & No & 0.79 & 0.95 & 75.1 \\
\hline 2 & $\begin{array}{c}\text { Frequency } \\
\text { accuracy } \\
(\mathrm{KHz})\end{array}$ & $\begin{array}{c}175.0 \\
00\end{array}$ & $\begin{array}{c}174.94 \\
9\end{array}$ & $\begin{array}{c}175.450 / 174.50 \\
0\end{array}$ & No & 174.975 & No & 0 & 0.85 & 0 \\
\hline 3 & $\begin{array}{c}\text { Frequency } \\
\text { error (W) }\end{array}$ & 1.5 & -1.5 & $2.0 /-2.0$ & No & 0.3 & No & 0 & 0.9 & 0 \\
\hline
\end{tabular}




\begin{tabular}{|c|c|c|c|c|c|c|c|c|c|c|}
\hline 4 & $\begin{array}{c}\text { Transmitti } \\
\text { ng power } \\
\text { (W) }\end{array}$ & 100.0 & 10.0 & $102 / 9$ & 9.45 & 9.5 & No & 0.5 & 0.9 & 45 \\
\hline 5 & $\begin{array}{c}\text { AM } \\
\text { modulatio } \\
\text { n factor } \\
(\%)\end{array}$ & 100 & 80 & $110 / 75$ & 78.75 & 79.0 & No & 0.2 & 0.85 & 14 \\
\hline 6 & $\begin{array}{c}\text { AM } \\
\text { amplitude } \\
\text { modulatio } \\
\text { ndistortion } \\
(\%)\end{array}$ & 6.0 & 0 & $6.5 / 0$ & 6.175 & 6.125 & No & 0.25 & 0.85 & 21.25 \\
\hline
\end{tabular}

The above data belongs to actual data measured by ATE equipment. Corresponding membership value is obtained through membership function operation. However, credibility factors are weighted, thereby obtaining fault prediction probability corresponding to all characteristic parameters. Finally, main factor outstanding fusion algorithm is adopted for obtaining $75.1 \%$ incentive module fault prediction probability. Namely, the equipment incentive module has 75.1 possible faults under current work condition. Actual equipment status tracking result shows that the method can be used for effectively obtaining current working states of all modules, and module fault probability can be predicted more accurately.

\section{Conclusion}

In the paper, the membership function method in fuzzy theory is adopted on the basis of working principle analysis and expert experience in some radio station. The fuzzy state of current equipment is quantified. Concrete data of the measured characteristic parameters is presented by membership value. Then, the above data is treated through introducing the credibility factor and related fusion algorithm, thereby finally achieving fault prediction of electronic equipment. It is discovered that the method is feasible and effective with higher prediction accuracy according to prediction result and continuous monitoring on equipment state.

\section{Acknowledgements}

This work was financially supported by the National Natural Science Fund (60874112).

\section{References}

[1] Xu Lijia, Wang Houjun, Long Bing. Complex system fault prediction based on Bayesian networks [J]. System Engineering and Electronics, Vol. 30: 780-784;

[3] Chen Shuili, Li Jinggong, etc. Fuzzy set theory and application thereof [M]. Beijing: Science Press, 2005;

[4] E Jiaqiang. Intelligent fault diagnosis and application thereof. Changsha: Hunan University Press, 2006;

[5] Ren Zhanyong. Aviation electronic product prediction and health management technology [M]. Beijing: National Defense Industry Press, 2013.3;

[6] He Yongshu, Chen Gan. Fault prediction analysis based on BP neural network model [J]. Microcomputer Information (Measurement and Control Automation) 2006.22: 220-222;

[7] Shi Wang, Sun Yufeng, Wang Zili, Zhao Guangyan. Analysis on PHM system and fault prediction model thereof [J]. Thermal Power and Command Control 2009(10), Vol. 34: 29-35; 